\section{L'hadronthérapie est une} modalité de radiothérapie qui utilise des faisceaux d'ions pour traiter des tumeurs, éventuellement proches d'organes à risque, en réduisant les effets secondaires dans les tissus sains. L'utilisation des ions carbone permet en plus d'atteindre des doses d'ionisation assez élevées pour traiter les cancers les plus radiorésistants.

Cette thérapie, fondée sur l'interaction d'ions rapides avec les tissus, fait appel au savoir-faire des physiciens. Cet article fait le bilan et la prospective du rôle de ces derniers dans le développement de la thérapie par ions carbone.

\title{
Les enjeux de l'hadronthérapie par ions carbone
}

Joseph Remillieux (1):*, Jean-Michel Moreau(2)* (jean-michel.moreau@univ-lyon1.fr), Denis Dauvergne ${ }^{(1) * *}$ et Jacques Balosso ${ }^{(3) * * * *}$

(1) Institut de Physique Nucléaire de Lyon (IPNL), Université Claude Bernard Lyon 1, UMR 5822 CNRS/IN2P3, 69622 Villeurbanne Cedex

(2) Laboratoire d'Informatique en Image et Systèmes d'information (LIRIS), Université Claude Bernard Lyon 1, UMR 5205 CNRS, 69622 Villeurbanne Cedex

(3) Institut des Neurosciences de Grenoble (GIN), INSERM U 836, CHU de Grenoble, Université Joseph Fourier, 38706 La Tronche Cedex

* Direction scientifique du Programme Rhônalpin de Recherche en Hadronthérapie pour ETOILE (PRRH)

** Direction du LabEx PRIMES et du Noud Rhône-Alpes Auvergne de France HADRON (FaraH)

***: Direction du GCS ETOllE et Direction de France HADRON.

L'ambition de doter la France d'un centre de traitement des tumeurs par faisceaux d'ions carbone est née, à la fin des années 1990, de la rencontre à l’Université Claude Bernard Lyon 1 de quelques médecins et physiciens, convaincus que la France avait toutes les capacités médicales et scientifiques pour initier un tel programme. Ce projet de " carbonethérapie " prit le nom de projet ETOILE (Espace de Traitement Oncologique par Ions Légers en Europe) au début des années 2000. Pour comprendre pourquoi il n'a pas été réalisé et évaluer les chances pour qu'il le soit un jour, il faut analyser les enjeux, les difficultés et les avantages de la carbonethérapie, comparée à la protonthérapie et aux formes les plus avancées de la radiothérapie.

\section{Les physiciens et le développement de l'hadronthérapie}

Le terme "hadronthérapie " est encore aujourd'hui énigmatique, tant pour les patients que pour la plupart des médecins. Ceci s'explique par le fait que c'est un physicien, R.R. Wilson [1], qui a suggéré l'intérêt qu'il pourrait y avoir dans le traitement des tumeurs solides à remplacer les " rayons " de la radiothérapie classique (des photons gamma, donc des bosons) par des faisceaux de protons (de la famille des hadrons).

Cette proposition, solidement étayée, ne suscita qu'un très faible intérêt chez les radiothérapeutes, mais l'enthousiasme unanime des physiciens. L'argument de

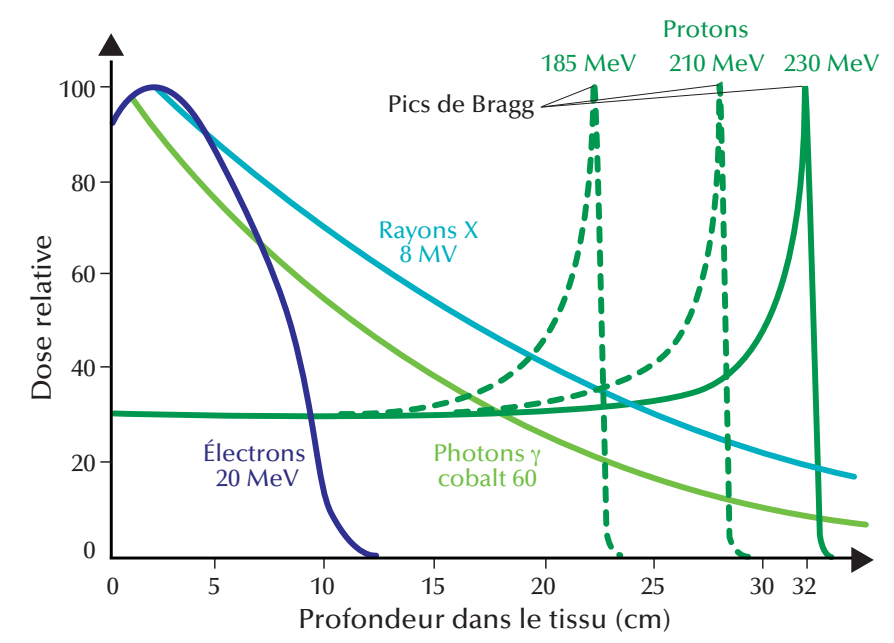

1. Le pic de Bragg. Représentation schématique du rendement de dose en profondeur d’un faisceau de protons mono-énergétiques, caractérisé par la présence d'un pic en fin de parcours (pic de Bragg), comparé à d'autres types de rayonnements utilisés en radiothérapie (photons $X$ ou électrons). La position du pic de Bragg dépend de l'énergie initiale des protons. D’après [3]. 


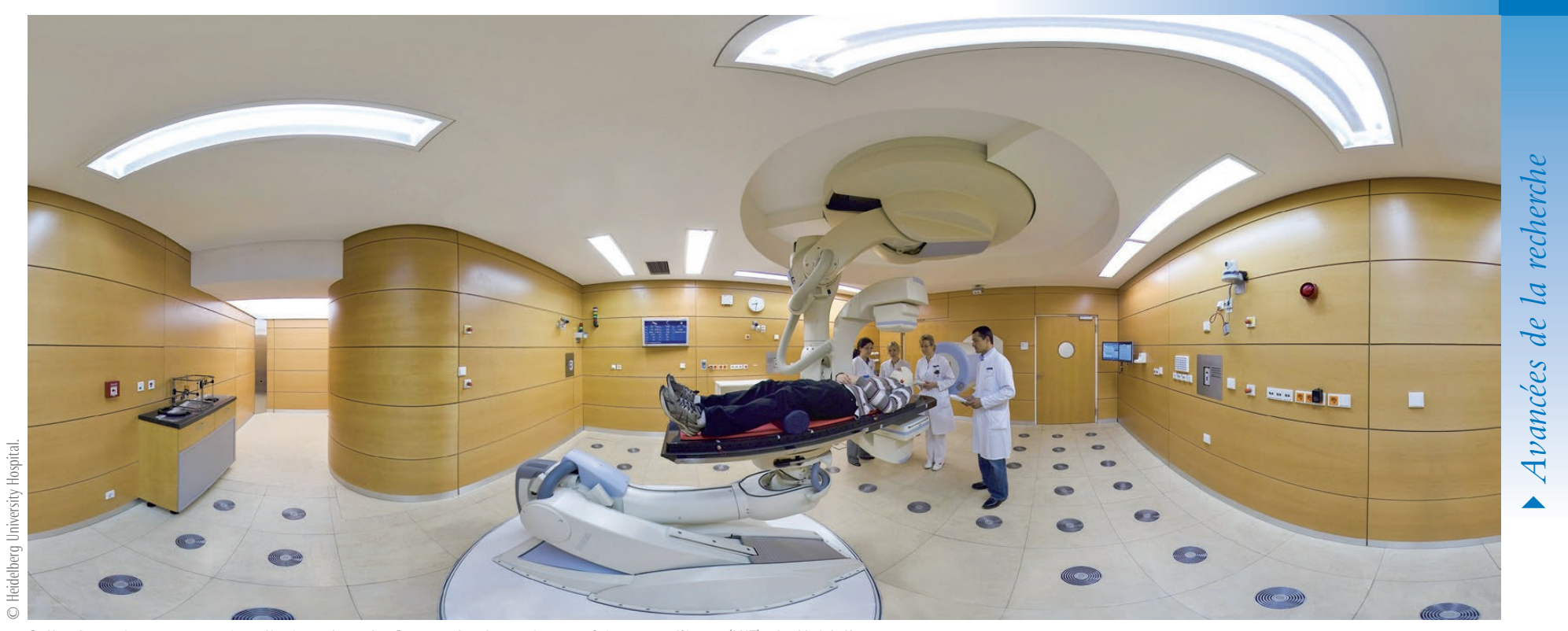

Salle de traitement par irradiation dans le Centre de thérapie par faisceaux d'ions (HIT) de Heidelberg.

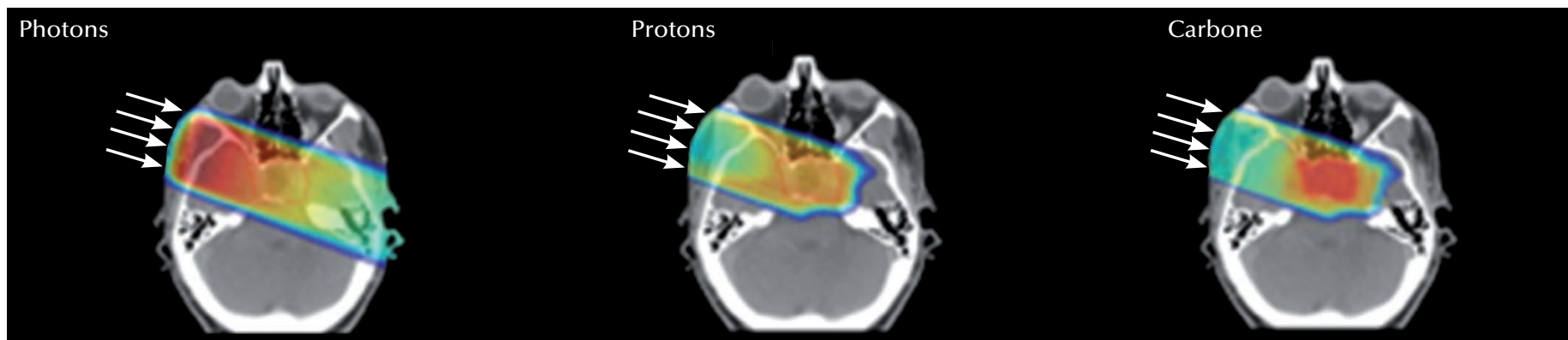

2. Simulation illustrative des profils de dépôts de dose (le rouge correspond aux doses les plus élevées) pour le traitement d'une tumeur cérébrale (au centre de chaque image, contour en orange) respectivement par des rayons X (à gauche), par des protons (au centre) et par des ions carbone (à droite). Source : GCS ETOILE.

Wilson était purement balistique, lié à l'existence du "pic de Bragg ", découvert par W.H. Bragg [2], montrant que c'est à la fin de leur parcours que les ions déposent le plus d'énergie par unité de longueur, et donc de densité locale d'ionisation, dans la matière qu'ils pénètrent (fig. 1).

Wilson proposait d'aligner un faisceau de protons dans la direction de la tumeur à traiter, puis d'ajuster l'énergie du faisceau pour que le parcours des protons corresponde rigoureusement à la profondeur de la tumeur. Cette balistique permet de disposer d'une efficacité maximum d'ionisation au sein de la tumeur pour stériliser les cellules cancéreuses, mais aussi de créer moins de dommages dans les tissus sains traversés en amont, tout en épargnant ceux qui sont situés au-delà de la zone d'arrêt des protons. Ces trois "avantages balistiques " sont à la base de l'intérêt potentiel de la "protonthérapie » [3] par rapport à la radiothérapie par rayons $\mathrm{X}$ traversant le corps du patient (fig. 2). La «visée » de la tumeur par le faisceau de protons ne pose pas de problème tant que la dispersion angulaire du faisceau au site de la tumeur reste faible ; cependant, la diffusion multiple des protons est d'autant moins négligeable que la tumeur est profonde.
De plus, le calcul du parcours des protons vers la tumeur se heurte à la complexité de la « cible " biologique traversée. Le ralentissement des ions dans la matière fut l'objet d'une succession de théories classiques puis quantiques et relativistes (N. Bohr 1913, H. Bethe 1930, F. Bloch 1933, U. Fano 1963...), et l'on travaille encore sur les raffinements de ces théories. Dans le cas de l'hadronthérapie, les distributions des compositions atomiques et des épaisseurs respectives des différents tissus traversés doivent être calculées à partir d'images de scanner $\mathrm{X}$ qui renseignent sur les taux d'absorption respectifs des rayons $\mathrm{X}$ dans ces tissus. La conversion de ces données d'absorption en énergie perdue par unité de longueur ou pouvoir d'arrêt $(d E / d x)$ des tissus pour les ions, encore appelé Transfert d'Énergie Linéique (TEL) par les biophysiciens, est complexe, d'autant plus que la cible est ici vivante, susceptible de se déplacer, voire de se déformer, pendant l'irradiation. Cette complexité, de nature mécanique et cinétique, est particulièrement difficile à surmonter pour les tumeurs associées à des organes très mobiles, tels que les poumons. Actuellement, les parcours et les dépôts de doses dans les tissus peuvent être simulés par des logiciels développés par les physiciens des particules pour concevoir leurs grands détecteurs, comme le code GEANT4. Il faut ensuite prévoir l'efficacité clinique de l'hadronthérapie dans la tumeur, ainsi que ses éventuels effets secondaires dans les tissus sains traversés, c'est-à-dire passer de la dose physique locale à la probabilité de survie des cellules. Cette étape est celle du radiobiologiste, qui étudie les effets primaires et secondaires des dépôts de doses, c'est-àdire les probabilités respectives d'endommagement, de réparation et de mort des cellules concernées. Dans la prévision et la simulation des effets cliniques, la collaboration entre biologistes et physiciens est indispensable, tant pour les aspects expérimentaux (dosimétrie) que théoriques (gerbes électroniques, radiolyse), et pour l'introduction des effets biologiques dans les codes de calcul.

Le savoir-faire des physiciens est aussi nécessaire pour que la séance d'irradiation puisse être guidée par l'image : leur expérience des grands détecteurs est transférable aux méthodes d'imagerie à développer pour le contrôle en temps réel de la distribution de la dose déposée dans l'organisme du patient. 
$>>$

L'hadronthérapie nécessite un accélérateur de particules permettant de traiter les tumeurs les plus profondes : pour pénétrer $30 \mathrm{~cm}$ de tissus il faut une énergie d'environ $220 \mathrm{MeV}$ avec des protons, et $400 \mathrm{MeV}$ par nucléon pour des ions carbone. C'est ainsi qu'en 1954, dans un laboratoire de physique nucléaire auprès du synchrotron Bevatron, au Lawrence Berkeley Laboratory, les premiers patients furent traités par protonthérapie. C'est dans ce même laboratoire que fut expérimentée dès 1975, auprès de l'accélérateur Bevalac, l'utilisation thérapeutique d'ions plus lourds que les protons (hélium, néon...). Cette innovation ouvrait l'hadronthérapie au domaine des hauts TEL, permettant d'atteindre dans le pic de Bragg des densités d'ionisation inaccessibles avec des rayonnements de bas TEL ( $\mathrm{X}$ et protons, cf. fig. 2 pour les ions carbone).

Les tissus cancéreux irradiés dans ces conditions de haut TEL étant stérilisés, même en cas d'hypoxie, il devenait possible de traiter des tumeurs très radiorésistantes, quasi intraitables autrement. Par ailleurs, les tissus sains traversés, en amont du pic de Bragg, ne subissaient pas plus de dommages que dans les thérapies à bas TEL. C'est à Berkeley qu'il fut ainsi démontré qu'un optimum thérapeutique était obtenu avec des ions carbone : minimum d'effets secondaires dans les tissus sains pour une dose permettant la stérilisation totale des tumeurs les plus radiorésistantes. La carbonethérapie était née. Afin de quantifier l'intérêt clinique de l'hadronthérapie, on introduit l'Efficacité Biologique Relative (EBR), qui est le rapport entre les doses respectives (en Gray, $1 \mathrm{G}=1 \mathrm{~J} / \mathrm{kg}$ ) de rayons $\mathrm{X}$ et d'ions conduisant au même taux de survie des cellules irradiées, c'est-à-dire au même effet biologique du traitement. L'expérience montre que l'EBR des ions carbone est de l'ordre de 3 , alors qu'il est proche de 1 pour les protons. Pour interpréter la haute efficacité biologique des ions carbone, les physiciens simulent les cascades d'électrons qui sont particulièrement denses dans leurs traces. Commence alors le travail collaboratif entre physiciens et radiobiologistes pour recenser, à toutes les échelles de temps, tous les effets primaires (les cassures multiples des brins de l'ADN) et secondaires (les effets physicochimiques tels que la radiolyse de l'eau) susceptibles d'être létaux pour les cellules atteintes. Ces effets doivent ensuite être introduits dans le code de calcul global pour établir le Plan de Traitement du patient.
Pour la carbonethérapie (et dans une moindre mesure la protonthérapie), ce calcul doit aussi intégrer la probabilité de fragmentation nucléaire des projectiles et des atomes cible, qui pollue le faisceau au cours de sa pénétration vers la tumeur (effet d'autant plus important que la tumeur est profonde). La simulation tient compte de la dispersion latérale des fragments, et de leur pénétration éventuelle au-delà du pic de Bragg, ce qui détériore la précision de la balistique de l'irradiation. En revanche, la fragmentation produit des rayonnements secondaires et des noyaux radioactifs qui permettent l'imagerie en ligne et le contrôle du traitement.

Pour résumer [4], la maîtrise totale de la carbonethérapie, vue par un physicien, repose sur l'établissement d'un code de calcul fondé sur les interactions électromagnétiques (parcours et cascades électroniques), les interactions fortes (émission de fragments nucléaires et rayonnements de desexcitation des noyaux), les interactions faibles (désintégration des fragments), l'étape physico-chimique (évolution des espèces chimiques réactives) et enfin l'étape radiobiologique (mort ou réparation des cellules dans les tissus irradiés). Un rôle central revient aussi au radiothérapeute, qui décide pour chaque patient de la pertinence du traitement, des doses à délivrer dans les différents volumes cibles, des niveaux acceptables d'incertitude, du fractionnement des irradiations et du suivi après traitement.

\section{Le développement des projets de carbonethérapie}

Il y a aujourd'hui près de 50 centres de protonthérapie dans le monde (dont la moitié en Amérique du Nord) et seulement une demi-douzaine de centres de soins opérationnels par ions carbone (Asie et Europe). En juin 2014, à la conférence PTCOG [5], on recensait 95424 patients traités par protonthérapie, et 12778 par ions carbone.

À la suite des premières expérimentations des physiciens aux États-Unis, deux pays se lancèrent dans la réalisation de centres de carbonethérapie : le Japon, qui ouvrit le premier centre (HIMAC, Chiba, 1994), et l'Allemagne, par le biais du GSI (Darmstadt, 1998), autre centre de recherche en physique, relayé en 2009 par le centre dédié HIT du CHU de Heidelberg (voit photo, p. 27). À ce jour, l'Allemagne a achevé la construction de deux centres
(Heidelberg et Marburg), et le second sera bientôt opérationnel. Le Japon compte cinq centres en service (Chiba1 1994, Hyogo 2001, Gunma 2010, Chiba2 2012, Saga-Tosu 2013), et un sixième est en cours de réalisation (Kanagawa). Le centre italien CNAO à Pavie a ouvert en 2011, avec un premier patient traité par ions carbone en septembre 2012. L'Autriche construit son centre (MedAustron) à Wiener-Neustadt, les premiers traitements par protons auront lieu en 2016. En Chine, outre un centre expérimental à Lanzhou, un des plus grands centres du monde vient d'ouvrir début 2014 à Shanghai.

La France comptait deux projets de centre carbone : le premier, historiquement, à Lyon (ETOILE, traitement, recherche clinique et $\mathrm{R} \& \mathrm{D}$ autour du patient) et le second à Caen (ARCHADE, recherche technologique sur de nouveaux types d'accélérateurs et expérimentation pour la carbonethérapie). À ce jour seul le projet ARCHADE est lancé, pour de la protonthérapie de routine et pour la mise au point d'un cyclotron cryogénique pour ions carbone.

Malgré sa validité clinique et technologique acquise pour le traitement de tumeurs radiorésistantes au Japon et en Allemagne, plusieurs raisons peuvent expliquer les difficultés rencontrées par les promoteurs de la carbonethérapie.

Du point de vue de la physique, d'abord : - les effets secondaires importants de la neutronthérapie, qui exploitait le potentiel thérapeutique associé au haut TEL des faisceaux de neutrons. Ces effets néfastes, liés à la mauvaise balistique associée à la pénétration des neutrons dans la matière, ont conduit à l'abandon progressif de la neutronthérapie (fermeture du centre d'Orléans en 2007). Cette expérience thérapeutique a cependant contribué à la compréhension des effets biologiques des hauts TEL dans les tissus, et la thérapie par capture neutronique par des noyaux ciblés tels que le bore reste une thérapie prometteuse [6] ;

- les faibles effets secondaires associés à la protonthérapie en font une méthode de soins particulièrement attractive en pédiatrie. Ceci explique en partie la grande attention portée aux centres de protonthérapie, notamment en France. 
Du point de vue économique et politique, ensuite :

- le processus décisionnel français a été affecté par l'absence de réalisation de centre de traitement par ions carbone aux États-Unis, pour des raisons liées au modèle économique de santé nord-américain. Les modèles de santé allemand, italien et autrichien, tous différents mais fondés sur une couverture sociale publique (ou via des compagnies d'assurances santé obligatoires), se sont révélés plus favorables ;

- la crainte des radiothérapeutes eux-mêmes de voir une partie du financement destiné à la carbonethérapie manquer à leur propre activité ;

- le retrait du principal acteur industriel européen de la carbonethérapie, Siemens, après la construction des centres de HIT et Marburg (et le démantèlement d'un troisième centre à Kiel, mais cependant la conduite à son terme du centre de Shanghai), a renforcé l'idée de nonrentabilité économique en Europe. Les centres italien et autrichien ont, quant à eux, bénéficié du savoir-faire et du soutien technologique du CERN. Pendant ce temps, les Japonais ont su associer les développements combinant recherche publique (NIRS) et partenaires industriels (Mitsubishi, Hitachi, Sumitomo et Toshiba);

- le coût des technologies et recherches associées à la carbonethérapie, qui peut être deux fois plus élevé que pour la protonthérapie, notamment pour la réalisation de dispositifs de distribution rotative du faisceau autour du patient.

\section{Les principaux axes de recherche en hadronthérapie}

Les principaux axes thématiques de recherche en hadronthérapie visent à optimiser la qualité, mais aussi le coût du traitement. Ces objectifs ont largement motivé le Programme régional de recherche en hadronthérapie (PRRH) autour du projet ETOILE de 2002 à 2013 [7], puis, de façon plus générale pour l'hadronthérapie par protons et ions carbone, au sein de France HADRON depuis 2012. (Voir aussi [4].)

1. Recherches médicales et médicoéconomiques. Il s'agit de déterminer les indications (fig. 3) nécessitant une thérapie par protons ou par ions carbone, à partir de modélisations, d'études cliniques et épidémiologiques, la mise en place
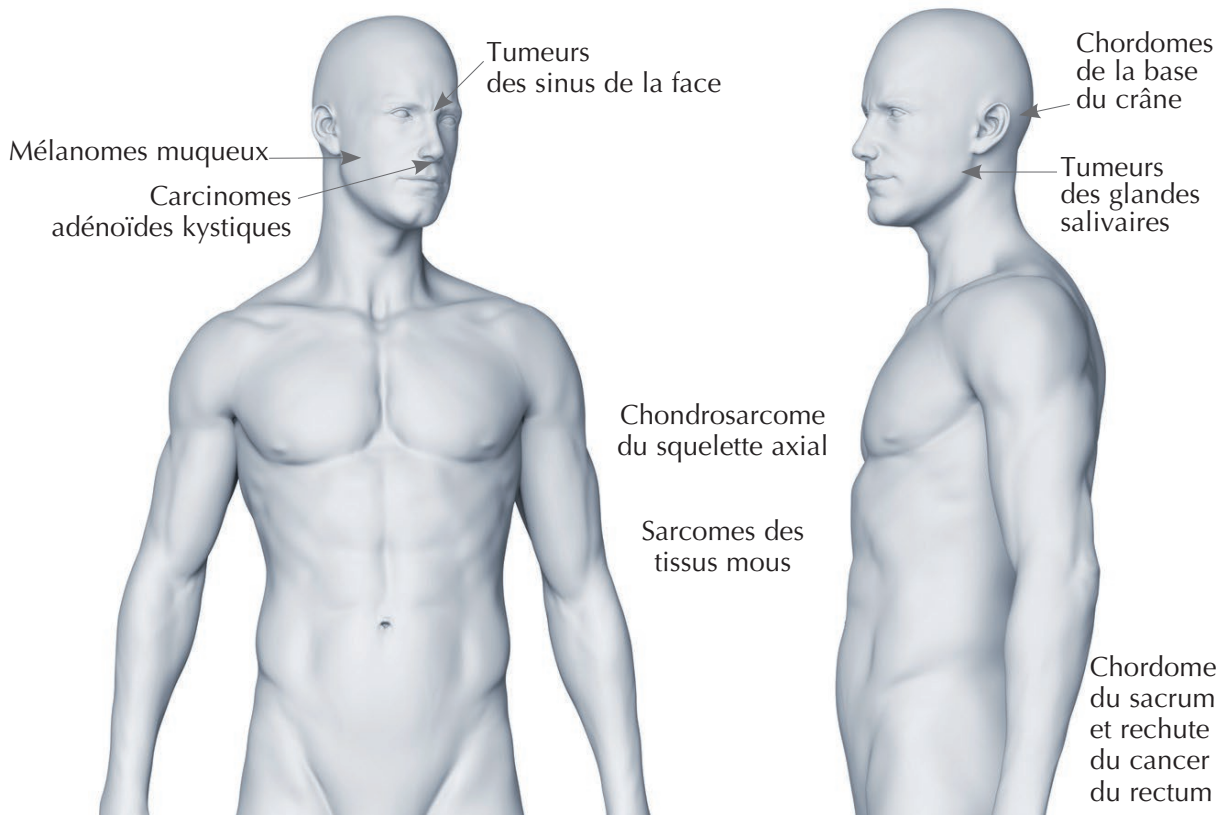

3. Indications consolidées pour la thérapie par ions carbone. Source : GS EtollE.

de réseaux de soins aux niveaux national et international et la détermination d'un flux de patients pouvant être traités dans un centre donné.

\section{Outils pour la planification du trai-} tement. Ces outils comprennent une modélisation in silico (informatique) des effets thérapeutiques, la modélisation physique du dépôt de dose, et l'optimisation des simulations pour le calcul du plan de traitement : certains paramètres nécessitent des volumes de calcul importants, qui entraînent un besoin croissant en puissance et en temps de calcul.

3. Radiobiologie. Les traitements par hadronthérapie s'appuient sur l'étude des effets radiobiologiques des ions sur les cellules saines ou tumorales, au niveau le plus fin (ADN) (fig. 4). Un problème important est de déterminer l'efficacité biologique relative (EBR) des différents types de rayonnements utilisés en radiothérapie. Ces informations se traduisent dans les modèles employés pour définir le plan de traitement de chaque tumeur identifiée.

4. Outils et instrumentation pour l'amélioration du traitement. En plus de développements spécifiques autour des accélérateurs et lignes de faisceaux (par exemple, le besoin d'irradier les tumeurs selon diverses incidences angulaires justifie l'étude de têtes rotatives isocentriques cryogéniques), les équipes françaises se concentrent sur : - le développement d'instruments pour le contrôle en ligne du traitement, permettant de connaitre et de contrôler en temps réel la dose distribuée et donc le traitement en cours de séance. Parmi les instrumentations développées figurent les capteurs de particules secondaires produites par les collisions nucléaires du projectile (gammas de désexcitation des noyaux, protons secondaires, ou encore fragments radioactifs pour la tomographie par émission de positons en ligne) ;

- le suivi des organes et tumeurs en mouvement. Pour pouvoir prendre en compte ces mouvements dans le cas d'une technique aussi performante que l'hadronthérapie, il est nécessaire de développer des méthodes de radiothérapie guidée par l'image et/ou fondées sur une modélisation biomécanique multi-échelle (de la cellule à l'organe).

\section{Conclusion et perspectives}

Dans le développement de la carbonethérapie, les laboratoires de physique ont joué un rôle fondamental : à Berkeley, au GSI (Darmstadt), et aussi au CERN via le projet R\&D PIMMS [8] et la fédération des efforts de recherche en hadronthérapie par le biais du consortium européen ENLIGHT. Cependant, les accélérateurs d'ions carbone restent encombrants, 
$>>$

onéreux, et délivrent des flux trop faibles pour certains traitements. On peut fonder beaucoup d'espoirs sur la récente décision du CERN d'investir dans l'hadronthérapie, tant sur le plan de la recherche technologique vers de nouveaux accélérateurs d'ions que sur la mise à disposition de lignes de faisceaux d'ions pour la radiobiologie des hauts TEL. De même, la récente décision de démarrer la R\&D pour un cyclotron supraconducteur à ARCHADE permet d'envisager des solutions sur le très long terme.

Du côté médical, le développement de la carbonethérapie recevra certainement une plus grande attention lorsque des études comparatives pourront mesurer de manière irréfutable l'apport de la radiothérapie à haut TEL pour le traitement des tumeurs radiorésistantes inopérables. Ces études sont en cours, notamment en Allemagne et en Italie. Elles ont pu démarrer pratiquement dès l'ouverture des centres européens, grâce à l'expérience accumulée par le NIRS (National Institute of Radiological Science) au Japon.

La contribution de la France passera par la collaboration et l'organisation d'études multicentriques telles que celle qui est soutenue par le Programme hospitalier de recherche clinique et la Caisse nationale d'assurance maladie, en collaboration avec le centre italien CNAO. C'est une des missions de l'Infrastructure nationale de recherche en santé et biotechnologie France HADRON, cofinancée par le programme Investissements d'avenir, de coordonner et entretenir une activité dynamique de recherche clinique et de $\mathrm{R} \& \mathrm{D}$ autour de la carbonethérapie, en attendant l'ouverture de centres français. Ceci doit être réalisé dans le cadre européen. On peut ainsi espérer que l'avance acquise ces dernières années ne soit pas perdue, alors que d'autres centres vont se construire ailleurs dans le monde, et notamment aux USA où plusieurs projets semi-publics se consolident progressivement sous l'égide du National Cancer Institute. Le rôle de projets européens comme ULICE (Union of Light Ion Centers in Europe, ENLIGHT) pour favoriser et dynamiser les collaborations scientifiques et médicales de recherche en hadronthérapie au sein de l'Europe est tout aussi important. I
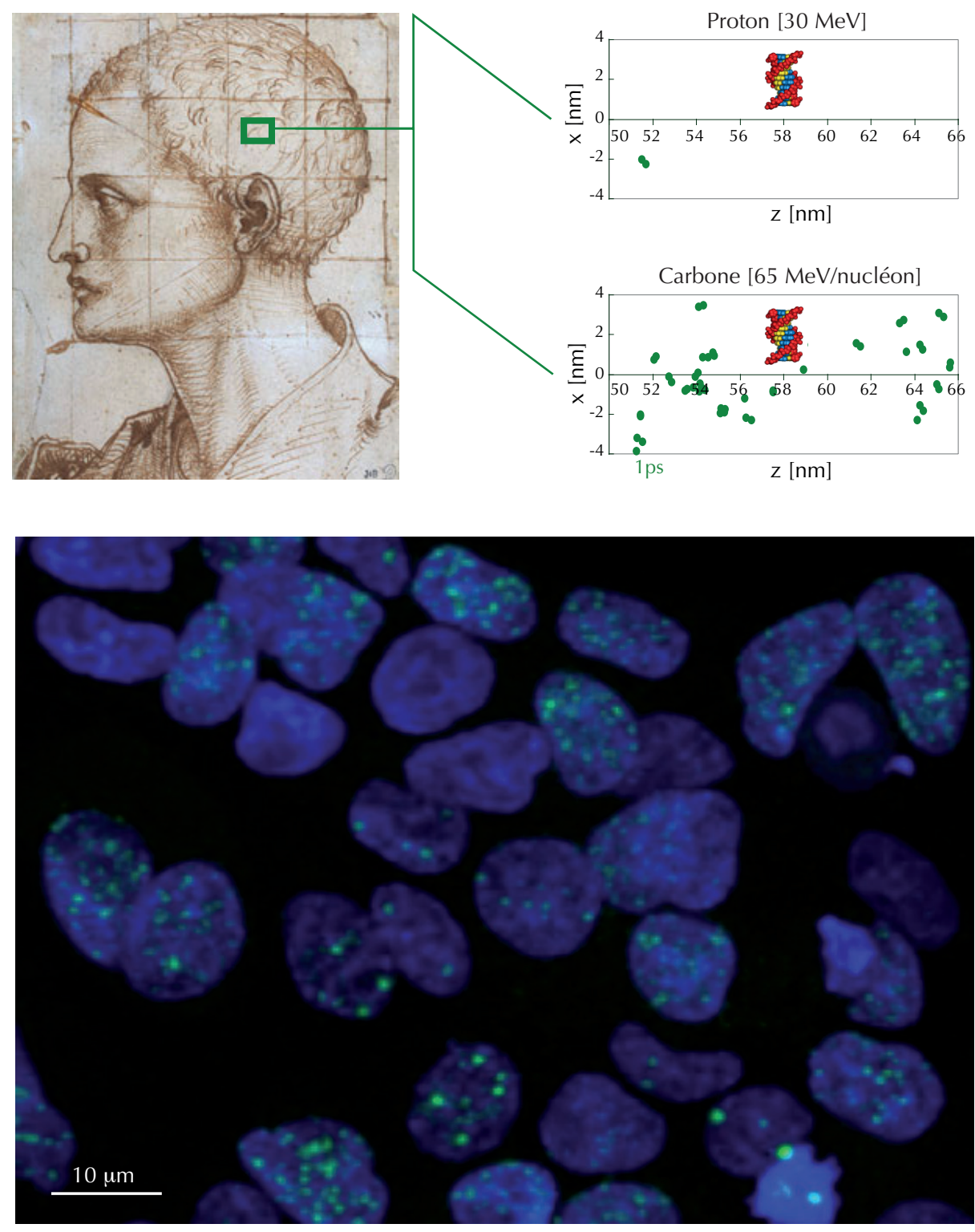

4. Radiobiologie.

Haut : production de radicaux libres (points verts), à la suite d'une irradiation par protons et ions carbone. Les particules se déplacent de la gauche vers la droite, le long de l’axe z.

Bas : image de microscopie par fluorescence. Les taches vertes matérialisent le marquage des cassures d'ADN double brin ; les zones bleues, le marquage du noyau des cellules. Source: PrRH/EOllE (PNN).

\section{Références}

1• R.R. Wilson, "Radiological uses of fast protons", Radiology 47 (1946) 487-491.

$2 \cdot W$. Bragg et R. Kleeman, " $0 n$ the $\alpha$ particles of radium, and their loss of range in passing through various atoms and molecules", Phil. Mag., 10 (1905) 318-340.

3 R. Ferrand, « La protonthérapie, technique de pointe à l'interface entre la physique et la médecine », Reflets de la physique 26 (2011) 4-9

4 The Nuclear Physics European Collaboration Committee, Nuclear Physics for Medicine (2014). www.nupecc.org/pub/npmed2014.pdf

5• www.ptcog.ch/archive/patient_statistics/Patientstatistics-updateMar2014.pdf

6• W.A.G. Sauerwein et al., Neutron Capture Therapy, Springer Verlag (2012).

7• M. Bajard et al., Rapport d'activité du Programme Régional de Recherche en Hadronthérapie pour le Centre ETOILE, Université Claude Bernard Lyon 1, juin 2013. URL: https://hal.archives-ouvertes.fr/hal-00840163.

8• L. Badano, P. Bryant et al., Proton-Ion Medical Machine Study (PIMMS), parts I and II, CERN/PS 99-010 and 00-007 DR (1999). 